\title{
ON RANDOM DISC POLYGONS IN SMOOTH CONVEX DISCS
}

\author{
F. FODOR, ${ }^{* *}$ University of Szeged and University of Calgary \\ P. KEVEI, ${ }^{* * *}$ MTA-SZTE Analysis and Stochastics Research Group \\ V. VÍGH, ${ }^{* * * * *}$ University of Szeged
}

\begin{abstract}
In this paper we generalize some of the classical results of Rényi and Sulanke (1963), (1964) in the context of spindle convexity. A planar convex disc $S$ is spindle convex if it is the intersection of congruent closed circular discs. The intersection of finitely many congruent closed circular discs is called a disc polygon. We prove asymptotic formulae for the expectation of the number of vertices, missed area, and perimeter difference of uniform random disc polygons contained in a sufficiently smooth spindle convex disc.
\end{abstract}

Keywords: Convex disc; disc polygon; spindle convexity; random approximation

2010 Mathematics Subject Classification: Primary 52A22

Secondary 60D05

\section{Introduction and results}

In their classical papers, Rényi and Sulanke [23]-[25] investigated the geometric properties of approximations of convex discs by random convex polygons. In particular, they considered the following probability model.

Let $K$ be a convex disc (a compact convex set with nonempty interior) in the Euclidean plane $\mathbb{R}^{2}$, and let $y_{1}, y_{2}, \ldots$ be independent random points chosen from $K$ according to the uniform probability distribution. Let $K_{n}$ denote the convex hull of $Y_{n}=\left\{y_{1}, \ldots, y_{n}\right\}$. The set $K_{n}$ is called a uniform random convex polygon in $K$. We use $\mathbb{E}(\cdot)$ to denote the expectation of a random variable in this probability model.

Rényi and Sulanke [23], [24] proved asymptotic formulae for the expectation of the number of vertices of $K_{n}$ and the expectation of the missed area of $K_{n}$ under the assumption that the boundary $\partial K$ of $K$ is three times continuously differentiable. They also proved an asymptotic formula for the expectation of the perimeter difference of $K$ and $K_{n}$ under stronger differentiability assumptions on $\partial K$ and assuming that the curvature $\kappa(x)>0$ for all $x \in \partial K$. For later comparison, we state their results below in a slightly modified form.

Let $f_{0}\left(K_{n}\right)$ denote the number of vertices of $K_{n}$, let $A(K)$ be the area of $K$, and let $\Gamma(\cdot)$ be Euler's gamma function. Then (cf. Satz 3 of [23, p. 83])

$$
\lim _{n \rightarrow \infty} \mathbb{E}\left(f_{0}\left(K_{n}\right)\right) n^{-1 / 3}=\sqrt[3]{\frac{2}{3 A(K)}} \Gamma\left(\frac{5}{3}\right) \int_{\partial K} \kappa(x)^{1 / 3} \mathrm{~d} x,
$$

Received 5 October 2012; revision received 5 February 2014.

* Postal address: Bolyai Institute, University of Szeged, Aradi vértanúk tere 1, H-6720 Szeged, Hungary.

** Email address: fodorf@math.u-szeged.hu

*** Email address: kevei@math.u-szeged.hu

**** Email address: vigvik@math.u-szeged.hu 
where integration is with respect to the one-dimensional Hausdorff measure on $\partial K$. We note that, with the help of Efron's identity [9], (1.1) directly implies the following statement:

$$
\lim _{n \rightarrow \infty} \mathbb{E}\left(A\left(K \backslash K_{n}\right)\right) n^{2 / 3}=\sqrt[3]{\frac{2 A(K)^{2}}{3}} \Gamma\left(\frac{5}{3}\right) \int_{\partial K} \kappa(x)^{1 / 3} \mathrm{~d} x .
$$

Rényi and Sulanke derived (1.2) by direct computation; cf. Formula (48) in Satz 1 of [24, p. 144].

Assuming that the boundary of $K$ is sufficiently smooth and $\kappa(x)>0$ for all $x \in \partial K$, Rényi and Sulanke proved the asymptotic formula

$$
\lim _{n \rightarrow \infty} \mathbb{E}\left(\operatorname{Per}(K)-\operatorname{Per}\left(K_{n}\right)\right) n^{2 / 3}=\frac{1}{12} \Gamma\left(\frac{2}{3}\right)(12 A(K))^{2 / 3} \int_{\partial K} \kappa(x)^{4 / 3} \mathrm{~d} x
$$

for the perimeter difference of $K$ and $K_{n}$; cf. formula (47) in Satz 1 of [24, p. 144].

For more information about approximations of convex bodies by random polytopes, we refer the reader to the recent book by Schneider and Weil [29], and the survey articles by Bárány [2], Schneider [28], and Weil and Wieacker [30].

In this article we investigate the $R$-spindle convex analogue of the above probability model. Let $R>0$. $R$-spindle convex discs are those convex discs that are intersections of (not necessarily finitely many) closed circular discs of radius $R$. For a precise definition of spindle convexity; see Section 2. The intersection of finitely many closed circular discs of radius $R$ is a closed convex $R$-disc polygon. Let $X$ be a compact set which is contained in a closed circular disc of radius $R$. The intersection of all $R$-spindle convex discs containing $X$ is called the $R$-spindle convex hull of $X$, and it is denoted by $\operatorname{conv}_{S, R}(X)$.

Now we are ready to define our probability model. Let $S$ be an $R$-spindle convex disc in $\mathbb{R}^{2}$. Let $x_{1}, x_{2}, \ldots$ be independent random points in $S$ chosen according to the uniform probability distribution (the Lebesgue measure in $S$ normalized by the area of $S$ ). The $R$-spindle convex hull $S_{n}^{R}=\operatorname{conv}_{s, R}\left(X_{n}\right)$, where $X_{n}=\left\{x_{1}, \ldots, x_{n}\right\}$, is called a uniform random $R$-disc polygon in $S$. We prove the $R$-spindle convex analogues of (1.1), (1.2), and (1.3) in this probability model.

The concept of spindle convexity was first introduced probably by Mayer [19] as a generalization of linear convexity in the wider context of Minkowski geometry. In the Euclidean plane $\mathbb{R}^{2}$, a closed convex set can be represented as an intersection of closed half-planes. In the definition of an $R$-spindle convex set, the radius $R$ closed circular discs play the role of closed half-planes. Thus, formally, the $R=\infty$ case corresponds to linear convexity.

Early investigations of spindle convex sets were carried out in the first half of the 20th century. For a short survey of the early history of the subject and references; see the paper by Danzer et al. [8]. Fejes Tóth proved packing and covering theorems for $R$-spindle convex discs in [12] and [13] in the 1980s. More recently, Bezdek et al. [5] and Kupitz et al. [17], [18] investigated spindle convex sets and proved numerous results about them, many of which are analogous to those of linearly convex sets. They also considered higher-dimensional $R$-spindle convex sets. Intersections of a finite number of radius $R$ closed balls in $\mathbb{R}^{d}$ are called ball polyhedra (cf. [5]). Such objects played important roles in the proofs of various results in the last 50 years; for a list; see [5]. Fodor and Vígh [14] proved asymptotic formulae for best approximations of $R$-spindle convex discs by $R$-disc polygons, generalizing some of the corresponding results of Fejes Tóth [11] and McClure and Vitale [20] about best approximations of linearly convex discs by convex polygons. There is a wealth of new information about properties of spindle convex bodies and ball polyhedra in the recent monographs by Bezdek [3], [4]. 
The notion of spindle convexity is related to diametrical completeness of convex bodies through the so-called spherical intersection property. A convex body $K$ is diametrically complete if, for any point $x \notin K$, the diameter of conv $(K \cup\{x\})$ is strictly larger than that of $K$. It was proved by Eggleston [10] that in a Banach space the diametrically complete convex bodies are exactly those which have the so-called spherical intersection property, that is, they are equal to the intersection of all closed balls whose centre is contained in $K$ and whose radius is equal to the diameter of $K$. In Euclidean spaces diametrically complete convex bodies are exactly those of constant width; however, in Minkowski spaces this is not the case. Recently, much effort has been devoted to investigating the properties of diametrically complete sets in Minkowski spaces where sets that are intersections of congruent closed balls play a fundamental role (see, for example, [22] and the references therein), and to investigating various properties of the ball hull; see, for example, [21] for more information.

Random approximations of $R$-spindle convex sets by $R$-disc polygons naturally appear, for example, in the so-called diminishing process of Bálint Tóth; see [1]. Let $D_{0}=B_{R}$ be the radius $R$ closed circular disc in $\mathbb{R}^{2}$ centred at the origin. Define the random process $\left(D_{n}, p_{n}\right)$ for $n \geq 1$ as follows. Let $p_{n+1}$ be a uniform random point in $D_{n}$, and let $D_{n+1}=D_{n} \cap\left(B_{R}+p_{n+1}\right)$. Then each $D_{n}$ is a (nonuniform random) $R$-disc polygon, and the process converges (in the Hausdorff metric of compact sets) to a set of constant width $R$ with probability 1 . This process can be readily generalized for a general convex body $K \subset \mathbb{R}^{d}$, in place of $B_{R}$, that contains the origin. If the body $K$ is symmetric with respect to the origin then it determines a Minkowski metric and the sets $K_{n}$ are all (random) spindle convex bodies with respect to $K$ in this Minkowski space.

Finally, we remark that there are various terms used for $R$-spindle convex sets in the literature. Mayer introduced the word 'Überkonvexität' in [19]. Authors of early articles used the translation of Mayer's term. Fejes Tóth [12], [13] named such sets ' $R$-convex'. Bezdek et al. [5] and Kupitz et al. [17], [18] used the expression 'spindle convex'. The notion of spindle convexity arose naturally and was investigated from different points of view, which explains the various names used for these sets and it also indicates their importance.

The main results of this article are described in the following theorems.

Theorem 1.1. Let $R>0$, and let $S$ be an $R$-spindle convex disc with $C^{2}$ smooth boundary and with the property that $\kappa(x)>1 / R$ for all $x \in \partial S$. Then

$$
\lim _{n \rightarrow \infty} \mathbb{E}\left(f_{0}\left(S_{n}^{R}\right)\right) n^{-1 / 3}=\sqrt[3]{\frac{2}{3 A(S)}} \Gamma\left(\frac{5}{3}\right) \int_{\partial S}\left(\kappa(x)-\frac{1}{R}\right)^{1 / 3} \mathrm{~d} x
$$

and

$$
\lim _{n \rightarrow \infty} \mathbb{E}\left(A\left(S \backslash S_{n}^{R}\right)\right) n^{2 / 3}=\sqrt[3]{\frac{2 A(S)^{2}}{3}} \Gamma\left(\frac{5}{3}\right) \int_{\partial S}\left(\kappa(x)-\frac{1}{R}\right)^{1 / 3} \mathrm{~d} x
$$

We note that the two statements are connected with an Efron-type relation [9]; see (5.10) in Section 5 . 
Theorem 1.2. Let $R>0$, and let $S$ be an $R$-spindle convex disc with $C^{5}$ smooth boundary and with the property that $\kappa(x)>1 / R$ for all $x \in \partial S$. Then

$$
\begin{aligned}
\lim _{n \rightarrow \infty} & \mathbb{E}\left(\operatorname{Per}(S)-\operatorname{Per}\left(S_{n}^{R}\right)\right) n^{2 / 3} \\
= & \frac{(12 A(S))^{2 / 3}}{36} \Gamma\left(\frac{2}{3}\right) \int_{\partial S}\left(\kappa(x)-\frac{1}{R}\right)^{1 / 3}\left(3 \kappa(x)+\frac{1}{R}\right) \mathrm{d} x .
\end{aligned}
$$

Theorem 1.3. Let $R>0$, and let $S=B_{R}$ be a circular disc of radius $R$. Then

$$
\begin{gathered}
\lim _{n \rightarrow \infty} \mathbb{E}\left(f_{0}\left(S_{n}^{R}\right)\right)=\frac{\pi^{2}}{2}, \\
\lim _{n \rightarrow \infty} \mathbb{E}\left(A\left(B_{R} \backslash S_{n}^{R}\right)\right) n=\frac{R^{2} \pi^{3}}{2},
\end{gathered}
$$

and

$$
\lim _{n \rightarrow \infty} \mathbb{E}\left(\operatorname{Per}\left(B_{R}\right)-\operatorname{Per}\left(S_{n}^{R}\right)\right) n=\frac{R \pi^{3}}{2} .
$$

It is somewhat surprising that the expectation of the number of vertices of uniform random spindle convex polygons in circular discs tends to a (very small) constant. Roughly speaking, this means that after choosing many random points from a circle, the spindle convex hull will have about five vertices. Note that this phenomenon has no analogue in linear convexity.

Furthermore, for a (linearly) convex disc $K$ with $C^{2}$ smooth boundary and strictly positive curvature, the asymptotic formulae (1.1) and (1.2) of Rényi and Sulanke follow from (1.4) and (1.5), respectively. Similarly, for a convex disc with $C^{5}$ smooth boundary and strictly positive curvature, the asymptotic formula (1.3) of Rényi and Sulanke follows from (1.6). Thus, the results of Theorems 1.1 and 1.2 are generalizations of the corresponding results of Rényi and Sulanke.

The rest of the paper is organized as follows. In Section 2 we introduce the necessary notation. In Section 3 we prove how the asymptotic formulae of Rényi and Sulanke follow from our results. In Section 4 we investigate some properties of disc caps of spindle convex discs that are used in the subsequent arguments. We give the proofs of Theorem 1.1 and Theorem 1.2 in Section 5. Finally, in Section 6 we provide an outline of the proof of Theorem 1.3.

\section{Definitions and notation}

In this paper we work in the Euclidean plane $\mathbb{R}^{2}$. We denote points of $\mathbb{R}^{2}$ by lowercase letters and sets of points by capitals, unless otherwise noted. For a point set $X \subset \mathbb{R}^{2}$, we write cl $X$ for the closure of $X$, int $X$ for the interior of $X, X^{\mathrm{C}}$ for the complement set of $X$, and $\partial X$ for the boundary of $X$. We use the notation $A(\cdot)$ and $\operatorname{Per}(\cdot)$ for the area and perimeter of compact sets in $\mathbb{R}^{2}$, respectively, while $\langle\cdot, \cdot\rangle$ denotes the usual Euclidean inner product in $\mathbb{R}^{2}$. The symbol $B_{R}$ denotes the closed circular disc of radius $R$ centred at the origin. We use $S_{R}^{1}$ to denote $\partial B_{R}$. We tacitly assume that the plane is embedded in $\mathbb{R}^{3}$, and write $x \times y$ for the cross product of the vectors $x$ and $y$. For two functions $f(n)$ and $g(n)$, we write $f(n) \sim g(n)$ if $\lim _{n \rightarrow \infty} f(n) / g(n)=1$. We also use the $O(\cdot)$ and $o(\cdot)$ notation throughout the article.

We say that the boundary of a convex disc $K$ is $C^{k}$ smooth if it is a $k$-times continuously differentiable simple closed curve in $\mathbb{R}^{2}$. We use the notation $\kappa(x)$ for the curvature of $\partial K$ at $x$. If the boundary of $K$ is $C^{2}$ smooth then at every $x \in \partial K$ there exists a unique outer unit normal vector $u_{x} \in S^{1}$ to $\partial K$. 
For a convex disc $K$, integration on the boundary of $K$ with respect to the one-dimensional Hausdorff measure (the arc length of $\partial K$ ) is denoted by $\int_{\partial K} \cdots \mathrm{d} x$. In the case that the boundary of $K$ is $C^{2}$ smooth and $f(u)$ is a measurable function on $S^{1}, \int_{S^{1}} f(u) \mathrm{d} u=\int_{\partial K} f\left(u_{x}\right) \kappa(x) \mathrm{d} x$ (cf. formula 2.5.30 of [27]).

Let $x, y \in \mathbb{R}^{2}$ be such that their distance does not exceed $2 R$. We define the closed $R$-spindle $[x, y]_{s, R}$ of $x$ and $y$ as the intersection of all closed circular discs of radius $R$ that contain both $x$ and $y$. The closed $R$-spindle of two points whose distance is greater than $2 R$ is defined to be the whole plane $\mathbb{R}^{2}$. A set $S \subseteq \mathbb{R}^{2}$ is called $R$-spindle convex if, from $x, y \in S$, it follows that $[x, y]_{s, R} \subseteq S$. Spindle convex sets are also convex in the usual linear sense. In this paper we restrict our attention to compact spindle convex sets. We call a compact set $S \subset \mathbb{R}^{2}$ with nonempty interior an $R$-spindle convex disc if it has the $R$-spindle convex property.

Below we list those properties of spindle convex discs that will be used in our arguments. For more detailed information about spindle convexity, we refer the reader to [5].

A compact convex set $S$ is $R$-spindle convex if and only if it is the intersection of (not necessarily finitely many) congruent closed circular discs of radius $R$ (cf. Corollary 3.4 of [5, p. 205]). If the closed circular disc $B_{R}+p$ contains an $R$-spindle convex disc $S$ and there is a point $x \in \partial S$ such that also $x \in \partial B_{R}+p$, then we say that $B_{R}+p$ supports $S$ at $x$. Let $P$ be a convex $R$-disc polygon, and let $B_{R}+p$ be a circle supporting $P$ at $H=\partial P \cap\left(\partial B_{R}+p\right)$. Then $H$ either consists of only one point, called a vertex, or it consists of the points of a closed circular arc, called a side (or edge) of $P$. The number of edges of $P$ equals the number of vertices of $P$ (except in the case that $P$ is a circle of radius $R$ ); we denote this number by $f_{0}(P)$.

If $S$ is an $R$-spindle convex disc with $C^{2}$ smooth boundary then $\kappa(x) \geq 1 / R$ for all $x \in \partial S$, and, for every unit vector $u \in S^{1}$, there exists a unique point $x \in \partial S$ such that $u=u_{x}$; we denote this point by $x_{u}$. We also note that if $x \in \partial S$ then $B_{R}+x-R u_{x}$ supports $S$ at $x$.

\section{The limiting case}

In this section we show how Theorems 1.1 and 1.2 imply the asymptotic formulae (1.1), (1.2), and (1.3) of Rényi and Sulanke.

Let $K$ be a (linearly) convex disc with $C^{2}$ smooth boundary, and let $\kappa(x)>0$ for all $x \in \partial K$. Let $\kappa_{\min }=\min _{\partial K} \kappa(x)>0$. It follows from Mayer's results (cf. (Ü4) and (Ü5) of [19, p. 521], or, for a more recent and more general reference; see also Theorem 2.5.4 of [27]) that $K$ is $R$-spindle convex for all $R \geq R_{0}=1 / \kappa_{\min }$. For $R \geq R_{0}$ and sufficiently large $n$, we introduce the notation

$$
\begin{aligned}
\delta_{S}^{R}(n) & =\mathbb{E}\left(A\left(K \backslash S_{n}^{R}\right)\right) n^{2 / 3}, \\
\delta(n) & =\mathbb{E}\left(A\left(K \backslash K_{n}\right)\right) n^{2 / 3}, \\
I_{S}^{R} & =\sqrt[3]{\frac{2 A^{2}}{3}} \Gamma\left(\frac{5}{3}\right) \int_{\partial K}\left(\kappa(x)-\frac{1}{R}\right)^{1 / 3} \mathrm{~d} x, \\
I & =\sqrt[3]{\frac{2 A^{2}}{3}} \Gamma\left(\frac{5}{3}\right) \int_{\partial K} \kappa^{1 / 3}(x) \mathrm{d} x,
\end{aligned}
$$

with $A=A(K)$.

We claim that (1.5) implies the asymptotic formula (1.2) of Rényi and Sulanke.

Let $\varepsilon>0$ be fixed. Then it follows from $\lim _{R \rightarrow \infty} I_{S}^{R}=I$ that there exists $R_{1}(\varepsilon)>R_{0}$ such 
that

$$
1-\varepsilon<\frac{I_{S}^{R}}{I}<1+\varepsilon
$$

for all $R>R_{1}(\varepsilon)$.

Elementary calculations show that there exists $R_{2}(\varepsilon) \geq R_{0}$, depending only on $K$ and $\varepsilon$ such that, for all $R>R_{2}(\varepsilon)$,

$$
\frac{A\left([p, q]_{s, R}\right)}{A\left([p, q]_{s, R_{0}}\right)-A\left([p, q]_{s, R}\right)}<\varepsilon
$$

for any points $p, q \in K$.

Let $D_{m}^{R}$ denote an $R$-disc polygon in $K$ with vertices $p_{1}, \ldots, p_{m}$ indexed in the cyclic order, and let $P_{m}$ denote the (linear) convex hull of $p_{1}, \ldots, p_{m}$. Note that this is a polygon with vertices $p_{1}, \ldots, p_{m}$. If $R>R_{2}(\varepsilon)$ then (3.2) yields

$$
1<\frac{\delta(n)}{\delta_{S}^{R}(n)}=1+\frac{\mathbb{E}\left(A\left(S_{n}^{R}\right)-A\left(K_{n}\right)\right)}{\mathbb{E}\left(A(K)-A\left(S_{n}^{R}\right)\right)}<1+\sup _{\substack{D_{m}^{R} \subset K \\ 2 \leq m \leq n}} \frac{A\left(D_{m}^{R}\right)-A\left(P_{m}\right)}{A\left(D_{m}^{R_{0}}\right)-A\left(D_{m}^{R}\right)}<1+\varepsilon .
$$

Now assume that $R>\max \left\{R_{1}(\varepsilon), R_{2}(\varepsilon)\right\}$. It is clear that, for any such $R$, it follows from the convergence $\lim _{n \rightarrow \infty} \delta_{S}^{R}(n) / I_{S}^{R}=1$ that there exists $n(R)$ such that

$$
1-\varepsilon<\frac{\delta_{S}^{R}(n)}{I_{S}^{R}}<1+\varepsilon
$$

for all $n \geq n(R)$. Thus, from (3.1), (3.3), (3.4), and

$$
\frac{\delta(n)}{I}=\frac{\delta(n)}{\delta_{S}^{R}(n)} \frac{\delta_{S}^{R}(n)}{I_{S}^{R}} \frac{I_{S}^{R}}{I}
$$

we obtain

$$
1-3 \varepsilon<\frac{\delta(n)}{I}<1+7 \varepsilon
$$

for all $R>\max \left\{R_{1}(\varepsilon), R_{2}(\varepsilon)\right\}$ and $n>n(R)$, which proves that

$$
\lim _{n \rightarrow \infty} \frac{\delta(n)}{I}=1 \text {. }
$$

A similar argument shows that (1.6) implies the asymptotic formula (1.3) of Rényi and Sulanke. Finally, (1.1) for the number of vertices follows by Efron's equality (5.10) below.

\section{Caps of spindle convex discs}

From now on we restrict our attention to the case in which $R=1$ and we omit $R$ from the notation. We use the simpler terms spindle convex and disc polygon in place of 1-spindle convex and 1-disc polygon, respectively. In particular, $B=B_{1}$ denotes the unit disc. The $R$-spindle convex analogues of the following lemmas can be obtained by simple scaling.

Let $S$ be a spindle convex disc with $C^{2}$ smooth boundary, and assume that $\kappa(x)>1$ for all $x \in \partial S$. A subset $D$ of $S$ is a disc cap of $S$ if $D=\operatorname{cl}\left(S \cap(B+p)^{\mathrm{C}}\right)$ for some point $p \in \mathbb{R}^{2}$. Note that in this case $\partial B+p$ intersects $\partial S$ in at most two points. (This follows, for example, from Theorem 2.5.4 of [27].) Thus, the boundary of a nonempty disc cap $D$ consists of at most two connected arcs: one arc is a subset of $\partial S$, and the other arc is a subset of $\partial B+p$. In order to define the vertex and the outer normal of a disc cap, we need the following claim. 
Lemma 4.1. Let $S$ be a spindle convex disc with $C^{2}$ smooth boundary, and assume that $\kappa(x)>$ 1 for all $x \in \partial S$. Let $D=\operatorname{cl}\left(S \cap(B+p)^{\mathrm{C}}\right)$ be a nonempty disc cap of $S$ (as above). Then there exists a unique point $x_{0} \in \partial S \cap \partial D$ such that there exists a $t \geq 0$ with $B+p=$ $B+x_{0}-(1+t) u_{x_{0}}$. We refer to $x_{0}$ as the vertex of $D$ and to $t$ as the height of $D$.

Proof. Pick any $x \in \partial S \cap \partial D$, and consider the vectors $\overrightarrow{p x}$ and the outer unit normal $u_{x}$. We claim that there is a unique $x$ for which $\overrightarrow{p x}$ is a positive multiple of $u_{x}$. The existence follows from a simple continuity argument since the angles formed by the two vectors have different orientations at the endpoints of $\partial S \cap \partial D$. Uniqueness is proved as follows. Suppose that both $x_{1} \neq x_{2}$ fulfil the requirements. Let $\varphi$ be the (positive) angle between $u_{x_{1}}$ and $u_{x_{2}}$, and denote by $I$ the arc of $\partial S$ between $x_{1}$ and $x_{2}$ (according to the positive orientation), and by $\Delta s$ the length of $I$. By the spindle convexity of $S, x_{1}$ and $x_{2}$ can be joined by a unit circular arc in $S$. The length of this circular arc is clearly smaller than $\Delta s$; on the other hand, it is larger than $\varphi$, and, thus, $\Delta s>\varphi$. Using the assumption that the curvature of $\partial S$ is strictly larger than 1 , we obtain

$$
\varphi=\int_{I} \kappa(s) \mathrm{d} s>\int_{I} \mathrm{~d} s=\Delta s>\varphi,
$$

a contradiction.

Let $D(u, t)$ denote the disc cap with vertex $x_{u} \in \partial S$ and height $t$. Note that, for each $u \in S^{1}$, there exists a maximal positive constant $t^{*}(u)$ such that $\left(B+x_{u}-(1+t) u\right) \cap S \neq \varnothing$ for all $t \in\left[0, t^{*}(u)\right]$. Let $V(u, t)=A(D(u, t))$, and let $\ell(u, t)$ denote the arc length of $\partial D(u, t) \cap\left(\partial B+x_{u}-(1+t) u\right)$.

Lemma 4.2. Let $S$ be a spindle convex disc with $C^{2}$ boundary such that $\kappa(x)>1$ for all $x \in \partial S$. Then, for a fixed $x \in \partial S$, the following assertions hold:

$$
\begin{aligned}
& \lim _{t \rightarrow 0^{+}} \ell\left(u_{x}, t\right) t^{-1 / 2}=2 \sqrt{\frac{2}{\kappa(x)-1}}, \\
& \lim _{t \rightarrow 0^{+}} V\left(u_{x}, t\right) t^{-3 / 2}=\frac{4}{3} \sqrt{\frac{2}{\kappa(x)-1}} .
\end{aligned}
$$

Proof. Assume that $x=(0,0)$ and $u_{x}=(0,-1)$. Then, in a sufficiently small open neighbourhood of the origin, $\partial S$ is the graph of a $C^{2}$ smooth function $f(\sigma)$. Taylor's theorem yields

$$
f(\sigma)=\frac{\kappa(x)}{2} \sigma^{2}+o\left(\sigma^{2}\right) \quad \text { as } \sigma \rightarrow 0 .
$$

In the same open neighbourhood of the origin, the boundary of $B+x-(1+t) u_{x}$ is the graph of the function $g_{t}(\sigma)=t+1-\sqrt{1-\sigma^{2}}$. Simple calculation yields the following positive solution of the equation $g_{t}(\sigma)=f(\sigma)$ :

$$
\sigma_{+}=\sqrt{\frac{2}{\kappa(x)-1}} t^{1 / 2}+o\left(t^{1 / 2}\right) \quad \text { as } t \rightarrow 0^{+} .
$$

Clearly, $\ell\left(u_{x}, t\right) \sim 2 \sigma_{+}$as $t \rightarrow 0^{+}$by the fact that the ratio of the lengths of an arc and the corresponding chord tends to 1 as the length of the arc tends to 0 . 
Let $\sigma_{-}$denote the negative solution of the equation $g_{t}(\sigma)=f(\sigma)$. Then

$$
\begin{aligned}
V\left(u_{x}, t\right) & =\int_{\sigma_{-}}^{\sigma_{+}}\left(g_{t}(\sigma)-f(\sigma)\right) \mathrm{d} \sigma \\
& =2 \int_{0}^{\sigma_{+}}\left[t+\frac{\sigma^{2}}{2}-\frac{\kappa\left(u_{x}\right)}{2} \sigma^{2}+o\left(\sigma^{2}\right)\right] \mathrm{d} \sigma \\
& =\frac{4}{3} \sqrt{\frac{2}{\kappa(x)-1}} t^{3 / 2}+o\left(t^{3 / 2}\right) \quad \text { as } t \rightarrow 0^{+} .
\end{aligned}
$$

This completes the proof.

Let $x_{1}, x_{2} \in S$ be two distinct points. Then there are exactly two disc caps of $S$, say $D_{-}\left(x_{1}, x_{2}\right)=\mathrm{cl}\left(S \cap\left(B+p_{-}\right)^{\mathrm{C}}\right)$ and $D_{+}\left(x_{1}, x_{2}\right)=\mathrm{cl}\left(S \cap\left(B+p_{+}\right)^{\mathrm{C}}\right)$ with the property that $x_{1}, x_{2} \in \partial B+p_{-}$and $x_{1}, x_{2} \in \partial B+p_{+}$. Let $V_{-}\left(x_{1}, x_{2}\right)=A\left(D_{-}\left(x_{1}, x_{2}\right)\right)$ and $V_{+}\left(x_{1}, x_{2}\right)=$ $A\left(D_{+}\left(x_{1}, x_{2}\right)\right)$, and assume that $V_{-}\left(x_{1}, x_{2}\right) \leq V_{+}\left(x_{1}, x_{2}\right)$.

Lemma 4.3. Let $S$ be a spindle convex disc with $C^{2}$ boundary, and assume that $\kappa(x)>1$ for all $x \in \partial S$. Then there exists a constant $\delta>0$, depending only on $S$, such that $V_{+}\left(x_{1}, x_{2}\right)>\delta$ for any two distinct points $x_{1}, x_{2} \in S$.

Proof. We note that $\left[x_{1}, x_{2}\right]_{s}$ cannot cover $S$ because of the $C^{2}$ smoothness of $\partial S$ and the assumption that $\kappa(x)>1$ for all $x \in \partial S$. Thus, by compactness, there exists a constant $\delta>0$, depending only on $S$, such that $A\left(S \backslash\left[x_{1}, x_{2}\right]_{s}\right)>2 \delta$ for any two distinct points $x_{1}, x_{2} \in S$. Now the statement of the lemma readily follows from the fact that $S=D_{-}\left(x_{1}, x_{2}\right) \cup D_{+}\left(x_{1}, x_{2}\right) \cup$ $\left[x_{1}, x_{2}\right]_{s}$.

Let $K$ be a convex disc with $C^{2}$ boundary and with the property that $\kappa(x)>0$ for all $x \in \partial K$. Let $\kappa_{0}>0$ denote the minimum of the curvature of $\partial K$. Then there exists an $\varepsilon_{0}>0$, depending only on $K$, with the property that, for any $x \in \partial K$, the (unique) circle of radius $1 / \kappa_{0}$ that is tangent to $\partial K$ at $x$ supports $K$ in a neighbourhood of radius $\varepsilon_{0}$ of $x$. Moreover, Mayer proved (see statement (Ü5) of [19, p. 521], or, for a more recent and more general reference; see also Theorem 2.5.4 of [27]) that in this case the tangent circles of radius $1 / \kappa_{0}$ of $\partial K$ not only locally support $K$ but also contain $K$ and, thus, they globally support $K$.

Let $S$ be a spindle convex disc with $C^{2}$ smooth boundary and with the property that $\kappa(x)>1$ for all $x \in \partial K$. Then, by the above, there exists $0<\hat{\varrho}<1$, depending only on $S$, such that $S$ has a supporting circular disc of radius $\hat{\varrho}$ at each $x \in \partial S$. Thus, it follows from Lemma 4.2 that there exists a $0<t_{0} \leq \hat{\varrho}$ with the property that, for any $u \in S^{1}$,

$$
\ell(u, t) \leq 4 \sqrt{\frac{2 \hat{\varrho}}{1-\hat{\varrho}}} t^{1 / 2} \quad \text { for } t \in\left[0, t_{0}\right]
$$

A convex disc $K$ has a rolling ball if there exists a real number $\varrho>0$ with the property that any $x \in \partial K$ lies in some closed circular disc of radius $\varrho$ contained in $K$. Hug [16] proved that the existence of a rolling ball is equivalent to the exterior unit normal being a Lipschitz function on $\partial K$. This implies that if the boundary of $K$ is $C^{2}$ smooth then $K$ has a rolling ball. We note that this last fact has already been observed by Blaschke [6].

It follows from the assumption that the boundary of $S$ is $C^{2}$ smooth that there exists a rolling ball for $S$ with radius $0<\varrho<1$. The existence of the rolling ball and (4.1) reveal that there 
exists $0<\hat{t}<\varrho$ such that, for any $u \in S^{1}$,

$$
V(u, t) \geq \frac{1}{2}\left(\frac{4}{3} \sqrt{\frac{2 \varrho}{1-\varrho}}\right) t^{3 / 2} \quad \text { for } t \in[0, \hat{t}] .
$$

Note that although the statements in Lemma 4.2 are not uniform in $u$, both (4.2) and (4.3) are uniform in $u$.

\section{Proofs of Theorem 1.1 and Theorem 1.2}

Proof of Theorem 1.1. We essentially use the method invented by Rényi and Sulanke [23]. Note that it is enough to prove the theorem for $R=1$, from which the statement follows by a scaling argument. Thus, from now on we assume that $R=1$, and omit $R$ in the notation.

Let $A=A(S)$. First observe that the pair of random points $x_{1}, x_{2}$ determine an edge of $S_{n}$ if and only if at least one of the disc caps $D_{-}\left(x_{1}, x_{2}\right)$ and $D_{+}\left(x_{1}, x_{2}\right)$ does not contain any other points from $X_{n}$. Thus,

$$
\mathbb{E}\left(f_{0}\left(S_{n}\right)\right)=\left(\begin{array}{l}
n \\
2
\end{array}\right) W_{n}
$$

where

$$
W_{n}=\frac{1}{A^{2}} \int_{S} \int_{S}\left[\left(1-\frac{V_{-}\left(x_{1}, x_{2}\right)}{A}\right)^{n-2}+\left(1-\frac{V_{+}\left(x_{1}, x_{2}\right)}{A}\right)^{n-2}\right] \mathrm{d} x_{1} \mathrm{~d} x_{2} .
$$

Note that if all points of $X_{n}$ fall into the closed spindle spanned by $x_{1}$ and $x_{2}$, then $x_{1}$ and $x_{2}$ contribute two edges to $S_{n}$ (since in this case $\operatorname{conv}_{\mathrm{s}} X_{n}=\left[x_{1}, x_{2}\right]_{s}$ ), and accordingly this event is counted in both terms in the integrand of (5.1).

Lemma 4.3 yields

$$
\begin{aligned}
\lim _{n \rightarrow \infty} & n^{-1 / 3}\left(\begin{array}{l}
n \\
2
\end{array}\right) \frac{1}{A^{2}} \int_{S} \int_{S}\left(1-\frac{V_{+}\left(x_{1}, x_{2}\right)}{A}\right)^{n-2} \mathrm{~d} x_{1} \mathrm{~d} x_{2} \\
& \leq \lim _{n \rightarrow \infty} n^{-1 / 3}\left(\begin{array}{l}
n \\
2
\end{array}\right) \frac{1}{A^{2}} \int_{S} \int_{S} \mathrm{e}^{-\delta(n-2) / A} \mathrm{~d} x_{1} \mathrm{~d} x_{2} \\
& =\lim _{n \rightarrow \infty} n^{-1 / 3}\left(\begin{array}{l}
n \\
2
\end{array}\right) \mathrm{e}^{-\delta(n-2) / A} \\
& =0 .
\end{aligned}
$$

Thus, the contribution of the second term of (5.1) is negligible; hence, in what follows, we will consider only the first term. Note that, by a similar argument, it is enough to integrate the first term of (5.1) over pairs of random points $x_{1}, x_{2}$ such that $V_{-}\left(x_{1}, x_{2}\right)<\delta$. Let $\mathbf{1}(\cdot)$ denote the indicator function of an event. Then

$$
\begin{aligned}
\lim _{n \rightarrow \infty} & \mathbb{E}\left(f_{0}\left(S_{n}\right)\right) n^{-1 / 3} \\
= & \lim _{n \rightarrow \infty} n^{-1 / 3}\left(\begin{array}{l}
n \\
2
\end{array}\right) \frac{1}{A^{2}} \int_{S} \int_{S}\left(1-\frac{V_{-}\left(x_{1}, x_{2}\right)}{A}\right)^{n-2} \mathbf{1}\left(V_{-}\left(x_{1}, x_{2}\right)<\delta\right) \mathrm{d} x_{1} \mathrm{~d} x_{2} .
\end{aligned}
$$

Now, we re-parametrize the pair $\left(x_{1}, x_{2}\right)$ as follows. Let

$$
\left(x_{1}, x_{2}\right)=\Phi\left(u, t, u_{1}, u_{2}\right),
$$


where $u, u_{1}, u_{2} \in S^{1}$ and $0 \leq t \leq t_{0}(u)$ are chosen such that

$$
D(u, t)=D_{-}\left(x_{1}, x_{2}\right)
$$

and

$$
\left(x_{1}, x_{2}\right)=\left(x_{u}-(1+t) u+u_{1}, x_{u}-(1+t) u+u_{2}\right) .
$$

Note that $u_{1}$ and $u_{2}$ are the unique outer unit normal vectors of $\partial B+x_{u}-(1+t) u$ at $x_{1}$ and $x_{2}$, respectively. Thus, for fixed $u$ and $t$, both $u_{1}$ and $u_{2}$ are in the same arc of length $\ell(u, t)$ in $S^{1}$. We denote this unit circular arc by $L(u, t)$.

Note that, since $V_{-}\left(x_{1}, x_{2}\right)<\delta, D_{-}\left(x_{1}, x_{2}\right)$ is uniquely determined by Lemma 4.3 . The uniqueness of the vertex and height of a disc cap guarantees that $\Phi$ is well defined, bijective, and differentiable (see Appendix A) on a suitable domain of $\left(u, t, u_{1}, u_{2}\right)$. To continue the estimate of $W_{n}$, we need the Jacobian of the transformation $\Phi$. This calculation can be found in [26], but, for the sake of completeness, we give a sketch in Appendix A.

The Jacobian of $\Phi$ satisfies

$$
|J \Phi|=\left(1+t-\frac{1}{\kappa\left(x_{u}\right)}\right)\left|u_{1} \times u_{2}\right| .
$$

We note that $\left|u_{1} \times u_{2}\right|$ equals the sine of the length of the unit circular arc between $x_{1}$ and $x_{2}$ on the boundary of $D(u, t)$. Also, note that there exists $t_{1}>0$ with the property that $V(u, t)<\delta$ for all $0 \leq t \leq t_{1}$ and all $u \in S^{1}$.

Equations (5.2) and (5.4) yield

$$
\begin{aligned}
\lim _{n \rightarrow \infty} & \mathbb{E}\left(f_{0}\left(S_{n}\right)\right) n^{-1 / 3} \\
=\lim _{n \rightarrow \infty} n^{-1 / 3}\left(\begin{array}{l}
n \\
2
\end{array}\right) \frac{1}{A^{2}} \int_{S^{1}} \int_{0}^{t_{1}} \int_{L(u, t)} \int_{L(u, t)} & \left(1-\frac{V(u, t)}{A}\right)^{n-2}\left(1+t-\frac{1}{\kappa\left(x_{u}\right)}\right) \\
& \times\left|u_{1} \times u_{2}\right| \mathrm{d} u_{1} \mathrm{~d} u_{2} \mathrm{~d} t \mathrm{~d} u .
\end{aligned}
$$

Integration by $u_{1}$ and $u_{2}$ yields

$$
\begin{aligned}
(5.5)=\lim _{n \rightarrow \infty} n^{-1 / 3}\left(\begin{array}{l}
n \\
2
\end{array}\right) \frac{2}{A^{2}} \int_{S^{1}} \int_{0}^{t_{1}} & \left(1-\frac{V(u, t)}{A}\right)^{n-2}\left(1+t-\frac{1}{\kappa\left(x_{u}\right)}\right) \\
& \times(\ell(u, t)-\sin \ell(u, t)) \mathrm{d} t \mathrm{~d} u .
\end{aligned}
$$

We will split the domain of integration with respect to $t$ into two parts. Let $h(n)=$ $(c \ln n / n)^{2 / 3}$, where $c$ is a positive (absolute) constant to be specified later. From (4.3), it follows that there exists $n_{0} \in \mathbb{N}$ and $\gamma_{1}>0$, depending only on $S$, such that if $n>n_{0}$ then $h(n)<t_{1}$, and $V(u, t)>\gamma_{1} h(n)^{3 / 2}$ for all $h(n) \leq t \leq t_{1}$ and all $u \in S^{1}$.

Lemma 5.1. Let $h(n)$ be defined as above. Then

$$
\begin{aligned}
\lim _{n \rightarrow \infty} n^{-1 / 3}\left(\begin{array}{l}
n \\
2
\end{array}\right) \frac{2}{A^{2}} \int_{S^{1}} \int_{h(n)}^{t_{1}} & \left(1-\frac{V(u, t)}{A}\right)^{n-2}\left(1+t-\frac{1}{\kappa\left(x_{u}\right)}\right) \\
& \times(\ell(u, t)-\sin \ell(u, t)) \mathrm{d} t \mathrm{~d} u=0 .
\end{aligned}
$$


Proof. Note that $t_{1} \leq 2 \pi$, and there exists a universal constant $\gamma_{2}>0$ such that $\ell(u, t)-$ $\sin \ell(u, t) \leq \gamma_{2}$ for all $0 \leq t \leq t_{1}$ and $u \in S^{1}$. Hence, for any fixed $u \in S^{1}$ and any $n>n_{0}$, it holds that

$$
\begin{aligned}
\int_{h(n)}^{t_{1}} & \left(1-\frac{V(u, t)}{A}\right)^{n-2}\left(1+t-\frac{1}{\kappa\left(x_{u}\right)}\right)(\ell(u, t)-\sin \ell(u, t)) \mathrm{d} t \\
& \leq 3 \gamma_{2} \int_{h(n)}^{t_{1}}\left(1-\frac{\gamma_{1} h(n)^{3 / 2}}{A}\right)^{n-2} \mathrm{~d} t \\
& \leq 3 \gamma_{2} \int_{0}^{t_{1}}\left(1-\frac{\gamma_{1} c(\ln n / n)}{A}\right)^{n-2} \mathrm{~d} t \\
& \leq 6 \gamma_{2} n^{-c \gamma_{1} / A} .
\end{aligned}
$$

Now, let $c>5 A /\left(3 \gamma_{1}\right)$. Then

$$
\begin{aligned}
\lim _{n \rightarrow \infty} n^{-1 / 3}\left(\begin{array}{l}
n \\
2
\end{array}\right) \frac{2}{A^{2}} \int_{S^{1}} \int_{h(n)}^{t_{1}} & \left(1-\frac{V(u, t)}{A}\right)^{n-2}\left(1+t-\frac{1}{\kappa\left(x_{u}\right)}\right) \\
& \times(\ell(u, t)-\sin \ell(u, t)) \mathrm{d} t \mathrm{~d} u \\
\leq & \gamma_{2} \frac{24 \pi}{A^{2}} \lim _{n \rightarrow \infty} n^{-1 / 3}\left(\begin{array}{l}
n \\
2
\end{array}\right) n^{-c \gamma_{1} / A} \\
& =0 .
\end{aligned}
$$

Now, for $n>n_{0}$, we define

$$
\begin{aligned}
\theta_{n}(u)=n^{-1 / 3}\left(\begin{array}{l}
n \\
2
\end{array}\right) \int_{0}^{h(n)} & \left(1-\frac{V(u, t)}{A}\right)^{n-2}\left(1+t-\frac{1}{\kappa\left(x_{u}\right)}\right) \\
& \times(\ell(u, t)-\sin \ell(u, t)) \mathrm{d} t
\end{aligned}
$$

and so

$$
\lim _{n \rightarrow \infty} \mathbb{E}\left(f_{0}\left(S_{n}\right)\right) n^{-1 / 3}=\lim _{n \rightarrow \infty} \frac{2}{A^{2}} \int_{S^{1}} \theta_{n}(u) \mathrm{d} u .
$$

Equation (11) of [7] states that, for any $\beta \geq 0, \omega>0$, and $\alpha>0$, we have

$$
\int_{0}^{g(n)} t^{\beta}\left(1-\omega t^{\alpha}\right)^{n} \mathrm{~d} t \sim \frac{1}{\alpha \omega^{(\beta+1) / \alpha}} \Gamma\left(\frac{\beta+1}{\alpha}\right) n^{-(\beta+1) / \alpha}
$$

as $n \rightarrow \infty$, assuming that

$$
\left(\frac{(\beta+\alpha+1) \ln n}{\alpha \omega n}\right)^{1 / \alpha}<g(n)<\omega^{-1 / \alpha}
$$

for sufficiently large $n$.

Formula (4.2) implies that there exists $\gamma_{3}>0$ such that $\ell(u, t)-\sin \ell(u, t)<\gamma_{3} t^{3 / 2}$ for all $0<t<t_{0}$ and $u \in S^{1}$. We recall that $1+t-1 / \kappa\left(x_{u}\right)<3$ for all $u \in S^{1}$ and $0 \leq t \leq t_{1}$. From (4.3) and (5.6) with $\alpha=\beta=\frac{3}{2}$ and $\omega=(2 /(3 A)) \sqrt{2 \rho /(1-\rho)}$ it follows that there exists $\gamma_{4}>0$, depending only on $S$, such that $\theta_{n}(u)<\gamma_{4}$ for all $u \in S^{1}$ and sufficiently large $n$. Thus, Lebesgue's dominated convergence theorem implies that

$$
\lim _{n \rightarrow \infty} \mathbb{E}\left(f_{0}\left(S_{n}\right)\right) n^{-1 / 3}=\frac{2}{A^{2}} \int_{S^{1}} \lim _{n \rightarrow \infty} \theta_{n}(u) \mathrm{d} u .
$$


Let $u \in S^{1}$ and $\varepsilon \in(0,1)$. It follows from Lemma 4.2 that there exists $0<t_{\varepsilon}<t_{1}$ such that

$$
(1-\varepsilon) \frac{4}{3}\left(\frac{2}{\kappa\left(x_{u}\right)-1}\right)^{3 / 2} t^{3 / 2} \leq \ell(u, t)-\sin \ell(u, t) \leq(1+\varepsilon) \frac{4}{3}\left(\frac{2}{\kappa\left(x_{u}\right)-1}\right)^{3 / 2} t^{3 / 2}
$$

and

$$
(1-\varepsilon) \frac{4}{3} \sqrt{\frac{2}{\kappa\left(x_{u}\right)-1}} t^{3 / 2} \leq V(u, t) \leq(1+\varepsilon) \frac{4}{3} \sqrt{\frac{2}{\kappa\left(x_{u}\right)-1}} t^{3 / 2}
$$

for any $t \in\left(0, t_{\varepsilon}\right)$.

From (5.7) and (5.8) we obtain

$$
\begin{aligned}
\lim _{n \rightarrow \infty} \theta_{n}(u)= & \frac{4 \sqrt{2}}{3}\left(\frac{1}{\kappa\left(x_{u}\right)-1}\right)^{3 / 2} \\
& \times\left[\frac{\kappa\left(x_{u}\right)-1}{\kappa\left(x_{u}\right)} \lim _{n \rightarrow \infty} n^{5 / 3} \int_{0}^{h(n)}\left(1-\frac{4}{3 A} \sqrt{\frac{2}{\kappa\left(x_{u}\right)-1}} t^{3 / 2}\right)^{n-2} t^{3 / 2} \mathrm{~d} t\right. \\
& \left.+\lim _{n \rightarrow \infty} n^{5 / 3} \int_{0}^{h(n)}\left(1-\frac{4}{3 A} \sqrt{\frac{2}{\kappa\left(x_{u}\right)-1}} t^{3 / 2}\right)^{n-2} t^{5 / 2} \mathrm{~d} t\right] .
\end{aligned}
$$

Note that (5.6) with $\alpha=\frac{3}{2}$ and $\beta=\frac{5}{2}$ implies that the second term of (5.9) is 0. Equation (5.6) yields

$$
\begin{gathered}
\lim _{n \rightarrow \infty} n^{5 / 3} \int_{0}^{h(n)}\left(1-\frac{4}{3 A} \sqrt{\frac{2}{\kappa\left(x_{u}\right)-1}} t^{3 / 2}\right)^{n-2} t^{3 / 2} \mathrm{~d} t \\
=\frac{2}{3}\left(\frac{4}{3 A} \sqrt{\frac{2}{\kappa\left(x_{u}\right)-1}}\right)^{-5 / 3} \Gamma\left(\frac{5}{3}\right) .
\end{gathered}
$$

Thus,

$$
\lim _{n \rightarrow \infty} \theta_{n}(u)=\frac{8 \sqrt{2}}{9}\left(\frac{1}{\kappa\left(x_{u}\right)-1}\right)^{3 / 2} \frac{\kappa\left(x_{u}\right)-1}{\kappa\left(x_{u}\right)}\left(\frac{4}{3 A} \sqrt{\frac{2}{\kappa\left(x_{u}\right)-1}}\right)^{-5 / 3} \Gamma\left(\frac{5}{3}\right) .
$$

Therefore,

$$
\begin{aligned}
\lim _{n \rightarrow \infty} \mathbb{E}\left(f_{0}\left(S_{n}\right)\right) n^{-1 / 3} & =\frac{2}{A^{2}} \int_{S^{1}} \lim _{n \rightarrow \infty} \theta_{n}(u) \mathrm{d} u \\
& =\sqrt[3]{\frac{2}{3 A}} \Gamma\left(\frac{5}{3}\right) \int_{S^{1}} \frac{1}{\kappa\left(x_{u}\right)}\left(\kappa\left(x_{u}\right)-1\right)^{1 / 3} \mathrm{~d} u \\
& =\sqrt[3]{\frac{2}{3 A}} \Gamma\left(\frac{5}{3}\right) \int_{\partial S}(\kappa(x)-1)^{1 / 3} \mathrm{~d} x .
\end{aligned}
$$

To compute the expectation of the missed area by $S_{n}$, we use the identity

$$
\mathbb{E}\left(f_{0}\left(S_{n}\right)\right)=\frac{n \mathbb{E}\left(A\left(S \backslash S_{n-1}\right)\right)}{A} .
$$


Equation (5.10) is the spindle convex analogue of Efron's identity [9], and is proved as follows:

$$
\begin{aligned}
\mathbb{E}\left(f_{0}\left(S_{n}\right)\right) & =\sum_{1}^{n} \mathbb{P}\left(x_{i} \text { is a vertex of } S_{n}\right) \\
& =n \mathbb{P}\left(x_{1} \text { is a vertex of } S_{n}\right) \\
& =n \mathbb{P}\left(x_{1} \notin \operatorname{conv}_{\mathrm{s}}\left(x_{2}, \ldots, x_{n}\right)\right) \\
& =\frac{n \mathbb{E}\left(A\left(S \backslash S_{n-1}\right)\right)}{A} .
\end{aligned}
$$

Combining (1.4) and (5.10) yields (1.5), thus completing the proof of Theorem 1.1.

We now turn to the proof of Theorem 1.2. The argument is based on ideas developed by Rényi and Sulanke [24], and it is similar to the argument used in the proof of Theorem 1.1.

We start with a refinement of Lemma 4.2 under the hypothesis that the boundary of $S$ is $C^{5}$ smooth and that $\kappa(x)>1$ for all $x \in \partial S$.

Lemma 5.2. Let $S$ be a spindle convex disc with $C^{5}$ smooth boundary and assume that $\kappa(x)>1$ for all $x \in \partial S$. Then, uniformly in $u \in S^{1}$,

$$
\begin{gathered}
\ell(u, t)=l_{1} t^{1 / 2}+l_{2} t^{3 / 2}+O\left(t^{5 / 2}\right) \quad \text { as } t \rightarrow 0^{+}, \\
\text {and } V(u, t)=v_{1} t^{3 / 2}+v_{2} t^{5 / 2}+O\left(t^{7 / 2}\right) \text { as } t \rightarrow 0^{+}
\end{gathered}
$$

with

$$
\begin{aligned}
l_{1} & =l_{1}(u)=2 \sqrt{\frac{2}{\kappa\left(x_{u}\right)-1}}, \\
l_{2}=l_{2}(u) & =\frac{2^{3 / 2}\left(15 b\left(x_{u}\right)^{2}-\left(\kappa\left(x_{u}\right)-1\right)\left(1+6\left(c\left(x_{u}\right)-1 / 8\right)-\kappa\left(x_{u}\right)\right)\right)}{3\left(\kappa\left(x_{u}\right)-1\right)^{7 / 2}}, \\
v_{1} & =v_{1}(u)=\frac{4}{3} \sqrt{\frac{2}{\kappa\left(x_{u}\right)-1}}, \\
v_{2} & =v_{2}(u)=\frac{2^{5 / 2}\left(5 b\left(x_{u}\right)^{2}-2\left(c\left(x_{u}\right)-1 / 8\right)\left(\kappa\left(x_{u}\right)-1\right)\right)}{5\left(\kappa\left(x_{u}\right)-1\right)^{7 / 2}},
\end{aligned}
$$

where $b(x)$ and $c(x)$ are functions depending only on $S$ and $x$.

Proof. With the same notation and choice of coordinate system as in the proof of Lemma 4.2, it follows from Taylor's theorem and the $C^{5}$ smoothness of the boundary that in a sufficiently small neighbourhood of the origin

$$
f(\sigma)=\frac{\kappa}{2} \sigma^{2}+b \sigma^{3}+c \sigma^{4}+O\left(\sigma^{5}\right) \quad \text { as } \sigma \rightarrow 0
$$

uniformly in $u \in S^{1}$. For brevity, we suppress the dependence of the coefficients on $u$. Let $g_{t}(\sigma)=t+1-\sqrt{1-\sigma^{2}}$. From the equation $f(\sigma)=g_{t}(\sigma)$ we obtain

$$
t=\frac{\kappa-1}{2} \sigma^{2}+b \sigma^{3}+\left(c-\frac{1}{8}\right) \sigma^{4}+O\left(\sigma^{5}\right) \quad \text { as } \sigma \rightarrow 0,
$$


and routine calculations reveal that the positive and negative solutions of the equation $f(\sigma)=$ $g_{t}(\sigma)$ are

$$
\begin{aligned}
& \sigma_{+}=\sigma_{+}(t)=d_{1} t^{1 / 2}+d_{2} t+d_{3} t^{3 / 2}+O\left(t^{2}\right) \quad \text { as } t \rightarrow 0^{+}, \\
& \sigma_{-}=\sigma_{-}(t)=-\left(d_{1} t^{1 / 2}-d_{2} t+d_{3} t^{3 / 2}\right)+O\left(t^{2}\right) \quad \text { as } t \rightarrow 0^{+},
\end{aligned}
$$

where

$$
d_{1}=\sqrt{\frac{2}{\kappa-1}}, \quad d_{2}=-\frac{2 b}{(\kappa-1)^{2}}, \quad d_{3}=\frac{\sqrt{2}\left(5 b^{2}-2(c-1 / 8)(\kappa-1)\right)}{(\kappa-1)^{7 / 2}} .
$$

Using the facts that $\ell(u, t)=\arcsin \sigma_{+}+\arcsin \left|\sigma_{-}\right|$and $V(u, t)=\int_{\sigma_{-}}^{\sigma_{+}}\left[g_{t}(\sigma)-f(\sigma)\right] \mathrm{d} \sigma, \mathrm{a}$ short calculation completes the proof.

Proof of Theorem 1.2. Let $L=\operatorname{Per}(S)$ for brevity. Let $x_{1}, x_{2} \in S$, and let $i\left(x_{1}, x_{2}\right)$ denote the length of the shorter unit circular arc joining $x_{1}$ and $x_{2}$. We define $U_{n}$ as

$$
\begin{aligned}
\mathbb{E}\left(\operatorname{Per}(S)-\operatorname{Per}\left(S_{n}\right)\right) & =L-\left(\begin{array}{l}
n \\
2
\end{array}\right) \mathbb{E}\left(\mathbf{1}\left(x_{1}, x_{2} \text { is an edge of } S_{n}\right) i\left(x_{1}, x_{2}\right)\right) \\
& =: L-\left(\begin{array}{l}
n \\
2
\end{array}\right) U_{n} .
\end{aligned}
$$

Using the same notation as in the proof of Theorem 1.1, similar arguments show that

$$
U_{n}=\frac{1}{A^{2}} \int_{S} \int_{S}\left[\left(1-\frac{V_{-}\left(x_{1}, x_{2}\right)}{A}\right)^{n-2}+\left(1-\frac{V_{+}\left(x_{1}, x_{2}\right)}{A}\right)^{n-2}\right] i\left(x_{1}, x_{2}\right) \mathrm{d} x_{1} \mathrm{~d} x_{2}
$$

and

$$
\lim _{n \rightarrow \infty} n^{2 / 3}\left(\begin{array}{l}
n \\
2
\end{array}\right) \frac{1}{A^{2}} \int_{S} \int_{S}\left(1-\frac{V_{+}\left(x_{1}, x_{2}\right)}{A}\right)^{n-2} i\left(x_{1}, x_{2}\right) \mathrm{d} x_{1} \mathrm{~d} x_{2}=0,
$$

and also that

$$
\begin{aligned}
& \lim _{n \rightarrow \infty} n^{2 / 3}\left(\begin{array}{l}
n \\
2
\end{array}\right) \frac{1}{A^{2}} \int_{S} \int_{S}\left(1-\frac{V_{-}\left(x_{1}, x_{2}\right)}{A}\right)^{n-2} \mathbf{1}\left(V_{-}\left(x_{1}, x_{2}\right)>\delta\right) i\left(x_{1}, x_{2}\right) \mathrm{d} x_{1} \mathrm{~d} x_{2} \\
& =0 .
\end{aligned}
$$

Now the integral transformation $\Phi$ in (5.3) yields

$$
\begin{array}{r}
\frac{1}{A^{2}} \int_{S} \int_{S}\left(1-\frac{V_{-}\left(x_{1}, x_{2}\right)}{A}\right)^{n-2} \mathbf{1}\left(V_{-}\left(x_{1}, x_{2}\right) \leq \delta\right) i\left(x_{1}, x_{2}\right) \mathrm{d} x_{1} \mathrm{~d} x_{2} \\
=\frac{1}{A^{2}} \int_{S^{1}} \int_{0}^{t_{1}} \int_{L(u, t)} \int_{L(u, t)}\left(1-\frac{V(u, t)}{A}\right)^{n-2}\left(1+t-\frac{1}{\kappa\left(x_{u}\right)}\right) \\
\times\left|u_{1} \times u_{2}\right| \arccos \left\langle u_{1}, u_{2}\right\rangle \mathrm{d} u_{1} \mathrm{~d} u_{2} \mathrm{~d} t \mathrm{~d} u,
\end{array}
$$

where $\arccos \left\langle u_{1}, u_{2}\right\rangle$ is the length of the arc of $S^{1}$ spanned by $u_{1}$ and $u_{2}$. Routine calculations show that

$$
\begin{aligned}
& \int_{L(u, t)} \int_{L(u, t)}\left|u_{1} \times u_{2}\right| \arccos \left\langle u_{1}, u_{2}\right\rangle \mathrm{d} u_{1} \mathrm{~d} u_{2} \\
& =2(2-2 \cos \ell(u, t)-\ell(u, t) \sin \ell(u, t)) .
\end{aligned}
$$


Let $\varepsilon>0$ be arbitrary. According to Lemma 5.2 we may choose $t_{2}>0$ such that, for all $t \in\left(0, t_{2}\right)$ and all $u \in S^{1}$,

$$
\left|\ell(u, t)-\left(l_{1} t^{1 / 2}+l_{2} t^{3 / 2}\right)\right| \leq \frac{\varepsilon}{2} t^{3 / 2}, \quad\left|V(u, t)-\left(v_{1} t^{3 / 2}+v_{2} t^{5 / 2}\right)\right| \leq \varepsilon t^{5 / 2} .
$$

For any $\varepsilon^{\prime}>0$ and sufficiently small $x$, it holds that

$$
\left|2(2-2 \cos x-x \sin x)-\left(\frac{x^{4}}{6}-\frac{x^{6}}{90}\right)\right| \leq \varepsilon^{\prime} x^{6},
$$

which, together with (5.11), implies that there exists $t_{3}>0$ with the property that, for any $t \in\left(0, t_{3}\right)$ and all $u \in S^{1}$,

$$
\left|2(2-2 \cos \ell(u, t)-\ell(u, t) \sin \ell(u, t))-\frac{1}{6}\left[l_{1}^{4} t^{2}+\left(4 l_{1}^{3} l_{2}-\frac{l_{1}^{6}}{15}\right) t^{3}\right]\right| \leq \frac{\varepsilon}{6} t^{3} .
$$

The second-order Taylor expansion of the function $\log (1-y)$ at $y=0$ implies that there exists $t_{4}>0$ such that, for $0<y \leq n \min _{u \in S^{1}} v_{1}(u) t_{4}^{2 / 3} / A$, any $c \in\left[-a_{1}, a_{1}\right]$, with $a_{1}=$ $A^{2 / 3} \max _{u \in S^{1}}\left|v_{2}(u) / v_{1}^{5 / 3}(u)\right|$, and all $u \in S^{1}$,

$$
\mathrm{e}^{-y} \mathrm{e}^{-(c+\varepsilon) y^{5 / 3} n^{-2 / 3}} \leq\left[1-\frac{y}{n}-c\left(\frac{y}{n}\right)^{5 / 3}\right]^{n} \leq \mathrm{e}^{-y} \mathrm{e}^{-c y^{5 / 3} n^{-2 / 3}}
$$

and

$$
\mathrm{e}^{-(1+\varepsilon) y} \leq\left[1-\frac{y}{n}-c\left(\frac{y}{n}\right)^{5 / 3}\right]^{n} \leq \mathrm{e}^{-(1-\varepsilon) y} .
$$

Let $\delta=\delta(\varepsilon)$ be small enough such that, for all $|y| \leq \delta$,

$$
\mathrm{e}^{-y} \leq 1-(1-\varepsilon) y
$$

and let $n_{0}$ be large enough such that

$$
\max _{u \in S^{1}} \frac{\left|v_{2}(u)\right| A^{2 / 3}}{v_{1}^{5 / 3}(u)} \leq n_{0}^{1 / 3} \delta .
$$

Finally, let $t^{\prime}:=\min \left\{t_{2}, t_{3}, t_{4}\right\}$. By a similar argument to that used in the proof of Lemma 5.1, we obtain

$$
\begin{aligned}
\lim _{n \rightarrow \infty} n^{2 / 3}\left(\begin{array}{l}
n \\
2
\end{array}\right) \frac{1}{A^{2}} \int_{S^{1}} \int_{t^{\prime}}^{t_{1}} & \left(1-\frac{V(u, t)}{A}\right)^{n-2} 2[2-2 \cos \ell(u, t)-\ell(u, t) \sin \ell(u, t)] \\
& \times\left(t+1-\frac{1}{\kappa\left(x_{u}\right)}\right) \mathrm{d} t \mathrm{~d} u
\end{aligned}
$$

$=0$.

Thus, we need to determine the limit

$$
\begin{aligned}
\lim _{n \rightarrow \infty} n^{2 / 3}\left[L-\left(\begin{array}{l}
n \\
2
\end{array}\right) \frac{1}{A^{2}} \int_{S^{1}} \int_{0}^{t^{\prime}}\right. & \left(1-\frac{V(u, t)}{A}\right)^{n} 2[2-2 \cos \ell(u, t)-\ell(u, t) \sin \ell(u, t)] \\
& \left.\times\left(t+1-\frac{1}{\kappa\left(x_{u}\right)}\right) \mathrm{d} t \mathrm{~d} u\right]
\end{aligned}
$$


By Lemma 5.2, for sufficiently small $t$, it holds uniformly in $u \in S^{1}$ that

$$
1 \leq\left(1-\frac{V(u, t)}{A}\right)^{-2} \leq 1+\frac{3 \max _{u \in S^{1}} v_{1}(u)}{A} t^{3 / 2} .
$$

Therefore, changing the exponent from $n-2$ to $n$ in the inner integral above does not affect either the main or the first-order term.

By (5.11) and (5.12), we have

$$
\begin{aligned}
\hat{\theta}_{n}(u):= & \frac{1}{A^{2}} \int_{0}^{t^{\prime}}\left(1-\frac{V(u, t)}{A}\right)^{n} 2[2-2 \cos \ell(u, t)-\ell(u, t) \sin \ell(u, t)]\left(t+1-\frac{1}{\kappa}\right) \mathrm{d} t \\
\leq & \frac{1}{6 A^{2}} \int_{0}^{t^{\prime}}\left(1-\frac{v_{1}}{A} t^{3 / 2}-\frac{v_{2}-\varepsilon}{A} t^{5 / 2}\right)^{n} \\
& \times\left[l_{1}^{4}\left(1-\frac{1}{\kappa}\right) t^{2}+\left(l_{1}^{4}+\left(1-\frac{1}{\kappa}\right)\left(4 l_{1}^{3} l_{2}-\frac{l_{1}^{6}}{15}\right)+\varepsilon\right) t^{3}\right] \mathrm{d} t .
\end{aligned}
$$

To ease the notation, let

$$
\begin{gathered}
D_{1}=l_{1}^{4}\left(1-\kappa^{-1}\right), \quad D_{1} D_{2}^{\varepsilon}=l_{1}^{4}+\left(1-\kappa^{-1}\right)\left(4 l_{1}^{3} l_{2}-\frac{l_{1}^{6}}{15}\right)+\varepsilon \\
\quad \text { and } \quad D_{2}=D_{2}^{0} .
\end{gathered}
$$

Letting $t^{\prime \prime}=\left(t^{\prime}\right)^{3 / 2} v_{1} / A$, the substitution $t^{3 / 2} v_{1} / A=y / n$ yields

$$
\begin{aligned}
\hat{\theta}_{n}(u) \leq \frac{D_{1}}{6 A^{2}} \int_{0}^{n t^{\prime \prime}}\left[1-\frac{y}{n}-\frac{v_{2}-\varepsilon}{A}\left(\frac{A y}{n v_{1}}\right)^{5 / 3}\right]^{n}\left(\frac{A y}{n v_{1}}\right)^{4 / 3} \\
\times\left[1+D_{2}^{\varepsilon}\left(\frac{A y}{n v_{1}}\right)^{2 / 3}\right] \frac{2}{3} y^{-1 / 3}\left(\frac{A}{n v_{1}}\right)^{2 / 3} \mathrm{~d} y \\
=\frac{D_{1}}{9 n^{2} v_{1}^{2}} \int_{0}^{n t^{\prime \prime}}\left[1-\frac{y}{n}-\frac{\left(v_{2}-\varepsilon\right) A^{2 / 3}}{v_{1}^{5 / 3}}\left(\frac{y}{n}\right)^{5 / 3}\right]^{n}\left[1+D_{2}^{\varepsilon}\left(\frac{A y}{n v_{1}}\right)^{2 / 3}\right] y \mathrm{~d} y \\
=: I_{n}+J_{n},
\end{aligned}
$$

where $I_{n}$ stands for the integral over the interval $\left[0, n^{1 / 5}\right]$, and $J_{n}$ stands for the integral over the interval $\left[n^{1 / 5}, t^{\prime \prime} n\right]$. Using (5.14), for $J_{n}$, we obtain

$$
J_{n} \leq \frac{D_{1}}{9 n^{2} v_{1}^{2}} \int_{n^{1 / 5}}^{n t^{\prime \prime}} \mathrm{e}^{-(1-\varepsilon) y} 2 n t^{\prime \prime} \mathrm{d} y \leq \frac{D_{1}}{9 v_{1}^{2}} \mathrm{e}^{-(1-\varepsilon) n^{1 / 5}},
$$

which tends to 0 faster than any polynomial of $n$. For $I_{n}$, using (5.13), (5.15), and (5.16) for $n \geq n_{0}$, we have

$$
\begin{aligned}
I_{n} & \leq \frac{D_{1}}{9 n^{2} v_{1}^{2}} \int_{0}^{n^{1 / 5}} \mathrm{e}^{-y} \exp \left\{-\frac{\left(v_{2}-\varepsilon\right) A^{2 / 3}}{v_{1}^{5 / 3}} \frac{y^{5 / 3}}{n^{2 / 3}}\right\}\left[1+D_{2}^{\varepsilon}\left(\frac{A y}{n v_{1}}\right)^{2 / 3}\right] y \mathrm{~d} y \\
& \leq \frac{D_{1}}{9 n^{2} v_{1}^{2}} \int_{0}^{n^{1 / 5}} \mathrm{e}^{-y}\left(1-(1-\varepsilon) \frac{\left(v_{2}-\varepsilon\right) A^{2 / 3}}{v_{1}^{5 / 3}} \frac{y^{5 / 3}}{n^{2 / 3}}\right)\left[1+D_{2}^{\varepsilon}\left(\frac{A y}{n v_{1}}\right)^{2 / 3}\right] y \mathrm{~d} y
\end{aligned}
$$




$$
\begin{aligned}
& \leq \frac{D_{1}}{9 n^{2} v_{1}^{2}} \int_{0}^{n^{1 / 5}} \mathrm{e}^{-y}\left[1+n^{-2 / 3} A^{2 / 3}\left(\frac{D_{2}^{\varepsilon}}{v_{1}^{2 / 3}} y^{2 / 3}-(1-\varepsilon) \frac{v_{2}-\varepsilon}{v_{1}^{5 / 3}} y^{5 / 3}+\varepsilon\right)\right] y \mathrm{~d} y \\
& \leq \frac{D_{1}}{9 n^{2} v_{1}^{2}}\left[1+n^{-2 / 3} A^{2 / 3}\left(\frac{D_{2}^{\varepsilon}}{v_{1}^{2 / 3}} \Gamma\left(\frac{8}{3}\right)-(1-\varepsilon) \frac{v_{2}-\varepsilon}{v_{1}^{5 / 3}} \Gamma\left(\frac{11}{3}\right)+2 \varepsilon\right)\right],
\end{aligned}
$$

where in the last inequality we extended the domain of the integration, and used the definition of the $\Gamma(\cdot)$ function.

We may obtain a lower estimate for $\hat{\theta}_{n}(u)$ in a similar way, and, as $\varepsilon>0$ was arbitrary, $\hat{\theta}_{n}(u)$ asymptotically equals the last upper bound with $\varepsilon=0$. Since $D_{1} /\left(18 v_{1}^{2}\right)=\kappa^{-1}$ and $\int_{S^{1}} \kappa^{-1}\left(x_{u}\right) \mathrm{d} u=L$, we have

$$
\begin{aligned}
\lim _{n \rightarrow \infty} \mathbb{E}\left(L-\operatorname{Per}\left(S_{n}\right)\right) n^{2 / 3} & =\lim _{n \rightarrow \infty} n^{2 / 3}\left(L-\left(\begin{array}{l}
n \\
2
\end{array}\right) \int_{S^{1}} \hat{\theta}_{n}(u) \mathrm{d} u\right) \\
& =\int_{S^{1}} \frac{D_{1} A^{2 / 3}}{18 v_{1}^{2}}\left(\frac{D_{2}}{v_{1}^{2 / 3}} \Gamma\left(\frac{8}{3}\right)-\frac{v_{2}}{v_{1}^{5 / 3}} \Gamma\left(\frac{11}{3}\right)\right) \mathrm{d} u .
\end{aligned}
$$

Substituting $D_{1}$ and $D_{2}$ from (5.17), and $l_{1}, l_{2}, v_{1}$, and $v_{2}$ from Lemma 5.2, we obtain

$$
\begin{aligned}
& \frac{D_{1} A^{2 / 3}}{18 v_{1}^{2}}\left(\frac{D_{2}}{v_{1}^{2 / 3}} \Gamma\left(\frac{8}{3}\right)-\frac{v_{2}}{v_{1}^{5 / 3}} \Gamma\left(\frac{11}{3}\right)\right) \\
& \quad=\frac{A^{2 / 3} \Gamma(8 / 3)}{\kappa} \frac{(3 / 2)^{2 / 3}\left[60 b^{2}+(\kappa-1)\left(5(\kappa-1)^{2}+9(\kappa-1)+3-24 c\right)\right]}{10(\kappa-1)^{8 / 3}},
\end{aligned}
$$

and, thus,

$$
\begin{aligned}
\lim _{n \rightarrow \infty} & \mathbb{E}\left(L-\operatorname{Per}\left(S_{n}\right)\right) n^{2 / 3} \\
& =\frac{(12 A)^{2 / 3} \Gamma(2 / 3)}{36} \int_{\partial S} \frac{(\kappa-1)\left(24 c-5(\kappa-1)^{2}-9(\kappa-1)-3\right)-60 b^{2}}{(\kappa-1)^{8 / 3}} \mathrm{~d} x .
\end{aligned}
$$

To complete the proof of Theorem 1.2, we must show that the constant in (5.18) is the same as in (1.6). Let $r(s)$ be the arc-length parametrization of $\partial S$. It is not difficult to verify that

$$
\begin{aligned}
& b(r(s))=\frac{1}{6}\left\langle r^{\prime \prime \prime}(s), \frac{r^{\prime \prime}(s)}{\kappa(r(s))}\right\rangle \\
& c(r(s))=\frac{1}{24}\left(\left\langle r^{(4)}(s), \frac{r^{\prime \prime}(s)}{\kappa(r(s))}\right\rangle-4 \kappa(r(s))\left\langle r^{\prime \prime \prime}(s), r^{\prime}(s)\right\rangle\right) .
\end{aligned}
$$

Upon substituting these formulae into (5.18), some tedious but straightforward calculations yield (1.6).

\section{The case of the unit circular disc}

In this section we discuss the case in which $S=B_{R}$. Note that in the hypotheses of Theorems 1.1 and 1.2 it is assumed that $\kappa(x)>1 / R$ for all $x \in \partial S$. This assumption no longer holds in the $S=B_{R}$ case, and, therefore, we may not use Lemma 4.3. However, the arguments used in the proofs of Theorems 1.1 and 1.2 can be modified slightly to yield a proof of Theorem 1.3. Below we provide the outline of the proof of Theorem 1.3 and leave the technical details to the interested reader. 
Proof of Theorem 1.3. As in the previous section, we may and do assume that $R=1$.

First note that, by Efron's identity (5.10), it is enough to prove (1.7) and (1.8). Also, note that, for any $u \in S^{1}$ and $0 \leq t \leq 2$, simple calculations yield

$$
\ell(u, t)=\ell(t)=2 \arcsin \sqrt{1-\frac{t^{2}}{4}}
$$

and

$$
V(u, t)=V(t)=t \sqrt{1-\frac{t^{2}}{4}}+2 \arcsin \frac{t}{2} .
$$

Let $W_{n}$ and $U_{n}$ be defined as in the proofs of Theorems 1.1 and 1.2, respectively, and let $L(t)=L(u, t)$ be defined as in the proof of Theorem 1.1. Then

$$
\begin{aligned}
W_{n} & =\frac{1}{\pi^{2}} \int_{S^{1}} \int_{0}^{2} \int_{L(t)} \int_{L(t)}\left(1-\frac{V(t)}{\pi}\right)^{n-2} t\left|u_{1} \times u_{2}\right| \mathrm{d} u_{1} \mathrm{~d} u_{2} \mathrm{~d} t \mathrm{~d} u, \\
U_{n} & =\frac{1}{\pi^{2}} \int_{S^{1}} \int_{0}^{2} \int_{L(t)} \int_{L(t)}\left(1-\frac{V(t)}{\pi}\right)^{n-2} t \arccos \left\langle u_{1}, u_{2}\right\rangle\left|u_{1} \times u_{2}\right| \mathrm{d} u_{1} \mathrm{~d} u_{2} \mathrm{~d} t \mathrm{~d} u .
\end{aligned}
$$

Integrating over $u_{1}, u_{2}$, and $u$ yields

$$
\begin{aligned}
& W_{n}=\frac{4}{\pi} \int_{0}^{2}\left(1-\frac{V(t)}{\pi}\right)^{n-2} t(\ell(t)-\sin \ell(t)) \mathrm{d} t \\
& U_{n}=\frac{4}{\pi} \int_{0}^{2}\left(1-\frac{V(t)}{\pi}\right)^{n-2} t(2-2 \cos \ell(t)-\ell(t) \sin \ell(t)) \mathrm{d} t .
\end{aligned}
$$

Upon substitution of (6.1), (6.2), and $t=2 \sin (\sigma / 2)$, we obtain

$$
\begin{aligned}
& W_{n}=\frac{4}{\pi} \int_{0}^{\pi} \sin \sigma(\pi-\sigma-\sin \sigma)\left(1-\frac{\sin \sigma+\sigma}{\pi}\right)^{n-2} \mathrm{~d} \sigma, \\
& U_{n}=\frac{4}{\pi} \int_{0}^{\pi} \sin \sigma(2+2 \cos \sigma-\sin \sigma(\pi-\sigma))\left(1-\frac{\sin \sigma+\sigma}{\pi}\right)^{n-2} \mathrm{~d} \sigma .
\end{aligned}
$$

By similar arguments as used in the proofs of Theorems 1.1 and 1.2, we obtain

$$
W_{n} \sim \frac{\pi^{2}}{n^{2}}, \quad U_{n} \sim \frac{4 \pi}{(n-2)^{2}}\left[1-\frac{1}{n-2}\left(\frac{\pi^{2}}{4}+3\right)\right]+O\left(n^{-3}\right),
$$

which yield the statements of Theorem 1.3.

\section{Appendix A}

In this section we sketch the calculation of the Jacobian of the transformation $\Phi$ defined in (5.3). We note that $J \Phi$ was calculated by Santaló [26].

Let $r:[0,2 \pi) \rightarrow \partial S$ be a parametrization of $\partial S$ such that the outer normal $u_{r(\alpha)}=$ $(\cos \alpha, \sin \alpha)$. We introduce $\alpha, \phi_{1}$, and $\phi_{2}$ such that $u=(\cos \alpha, \sin \alpha), u_{1}=\left(\cos \phi_{1}, \sin \phi_{1}\right)$, and $u_{2}=\left(\cos \phi_{2}, \sin \phi_{2}\right)$. Clearly, $\mathrm{d} u \mathrm{~d} u_{1} \mathrm{~d} u_{2}=\mathrm{d} \alpha \mathrm{d} \phi_{1} \mathrm{~d} \phi_{2}$. 
To make the calculation more apparent, we add an extra step: let $(v, w)$ be the centre of the unit circle that defines $D_{-}\left(x_{1}, x_{2}\right)$ (here $\left.v, w \in \mathbb{R}\right)$. Then $x_{1}=\left(v+\cos \phi_{1}, w+\sin \phi_{1}\right)$ and $x_{2}=\left(v+\cos \phi_{2}, w+\sin \phi_{2}\right)$, and by differentiation we obtain

$$
\mathrm{d} x_{1} \mathrm{~d} x_{2}=\left|\left(\sin \phi_{1} \cos \phi_{2}-\sin \phi_{2} \cos \phi_{1}\right)\right| \mathrm{d} \phi_{1} \mathrm{~d} \phi_{2} \mathrm{~d} v \mathrm{~d} w .
$$

Next observe that $(v, w)=\left(r_{1}(\alpha)-(1+t) \cos \alpha, r_{2}(\alpha)-(1+t) \sin \alpha\right)$; thus,

$$
\mathrm{d} v \mathrm{~d} w=\left|\left(-r_{1}^{\prime}(\alpha) \sin \alpha+r_{2}^{\prime}(\alpha) \cos \alpha-(1+t)\right)\right| \mathrm{d} \alpha \mathrm{d} t,
$$

and, hence,

$$
\mathrm{d} x_{1} \mathrm{~d} x_{2}=\left|\left(-r_{1}^{\prime}(\alpha) \sin \alpha+r_{2}^{\prime}(\alpha) \cos \alpha-(1+t)\right) \sin \left(\phi_{1}-\phi_{2}\right)\right| \mathrm{d} \phi_{1} \mathrm{~d} \phi_{2} \mathrm{~d} \alpha \mathrm{d} t .
$$

Using the special choice of $r(\alpha)$, we see that $-r_{1}^{\prime}(\alpha) \sin \alpha+r_{2}^{\prime}(\alpha) \cos \alpha=1 / \kappa(r(\alpha))$, and, by assumption, $\kappa>1$; thus,

$$
\left|\left(-r_{1}^{\prime}(\alpha) \sin \alpha+r_{2}^{\prime}(\alpha) \cos \alpha-(1+t)\right) \sin \left(\phi_{1}-\phi_{2}\right)\right|=\left(1+t-\frac{1}{\kappa(r(\alpha))}\right) \sin \left(\left|\phi_{1}-\phi_{2}\right|\right) \text {. }
$$

We note that $\left|u_{1} \times u_{2}\right|$ equals the sine of the length of the unit circular arc between $x_{1}$ and $x_{2}$ on the boundary of $D(u, t)$, that is, $\sin \left(\left|\phi_{1}-\phi_{2}\right|\right)=\left|u_{1} \times u_{2}\right|$, which proves (5.4).

\section{Acknowledgements}

The authors thank the anonymous referee for suggestions that greatly improved the manuscript.

The first author was supported by the János Bolyai Research Scholarship of the Hungarian Academy of Sciences, and by the Hungarian-Mexican Intergovernmental S\&T Cooperation Programmes TÉT_10-1-2011-0471 and NIH B330/479/11 Discrete and Convex Geometry. The first and third authors were also supported by the Hungarian Scientific Research Fund OTKA grant 75016, and by the Fields Institute for Research in Mathematical Sciences.

The research of the second and third authors was supported by the European Union and the State of Hungary, co-financed by the European Social Fund in the framework of TÁMOP 4.2.4. A/2-11-1-2012-0001 National Excellence Program. The second author was supported by the Hungarian Scientific Research Fund OTKA PD106181.

\section{References}

[1] Ambrus, G., Kevei, P. And Vígh, V. (2012). The diminishing segment process. Statist. Prob. Lett. 82, 191-195.

[2] Bárány, I. (2008). Random points and lattice points in convex bodies. Bull. Amer. Math. Soc. (N.S.) 45, 339-365.

[3] Bezdek, K. (2010). Classical Topics in Discrete Geometry. Springer, New York.

[4] BezdeK, K. (2013). Lectures on Sphere Arrangements-The Discrete Geometric Side (Fields Inst. Monogr. 32). Springer, New York.

[5] Bezdek, K., Lángi, Z., Naszódi, M. and Papez, P. (2007). Ball-polyhedra. Discrete Comput. Geom. 38, 201-230.

[6] BlaschKe, W. (1956). Kreis und Kugel. Walter de Gruyter, Berlin.

[7] Böröczky, K. J., Fodor, F., Reitzner, M. AND VíGH, V. (2009). Mean width of random polytopes in a reasonably smooth convex body. J. Multivariate Anal. 100, 2287-2295.

[8] Danzer, L., Grünbaum, B. And Klee, V. (1963). Helly's theorem and its relatives. In Proc. Sympos. Pure Math., Vol. VII, American Mathematical Society, Providence, RI, pp. 101-180.

[9] Efron, B. (1965). The convex hull of a random set of points. Biometrika 52, 331-343.

[10] EgGleston, H. G. (1965). Sets of constant width in finite dimensional Banach spaces. Israel J. Math. 3, 163-172. 
[11] Fejes Tóth, L. (1953). Lagerungen in der Ebene, auf der Kugel und im Raum. Springer, Berlin.

[12] Fejes Tóth, L. (1982). Packing of $r$-convex discs. Studia Sci. Math. Hungar. 17, 449-452.

[13] Fejes Tóth, L. (1982). Packing and covering with $r$-convex discs. Studia Sci. Math. Hungar. 18, 69-73.

[14] Fodor, F. AND Vígh, V. (2012). Disc-polygonal approximations of planar spindle convex sets. Acta Sci. Math. (Szeged) 78, 331-350.

[15] Gruber, P. M. (1997). Comparisons of best and random approximation of convex bodies by polytopes. II International Conference in 'Stochastic Geometry, Convex Bodies and Empirical Measures' (Agrigento, 1996). Rend. Circ. Mat. Palermo (2) Suppl. 50, 189-216.

[16] Hug, D. (1999). Measures, curvatures and currents in convex geometry. Habilitationsschrift, Albert Ludwigs Universität Freiburg.

[17] Kupitz, Y. S., Martini, H. And Perles, M. A. (2005). Finite sets in $\mathbb{R}^{d}$ with many diameters - a survey. In Proceedings of the International Conference on Mathematics and Applications (ICMA-MU 2005, Bangkok), Mahidol University Press, Bangkok, pp. 91-112.

[18] Kupitz, Y. S., Martini, H. And Perles, M. A. (2010). Ball polytopes and the Vázsonyi problem. Acta Math. Hungar. 126, 99-163.

[19] Mayer, A. E. (1935). Eine Überkonvexität. Math. Z. 39, 511-531.

[20] McClure, D. E. and Vitale, R. A. (1975). Polygonal approximation of plane convex bodies. J. Math. Anal. Appl. 51, 326-358.

[21] Moreno, J. P. AND Schneider, R. (2007). Continuity properties of the ball hull mapping. Nonlinear Anal. 66, 914-925.

[22] Moreno, J. P. And Schneider, R. (2012). Diametrically complete sets in Minkowski spaces. Israel J. Math. 191, 701-720.

[23] RÉnYI, A. And Sulanke, R. (1963). Über die konvexe Hülle von $n$ zufällig gewählten Punkten. $Z$. Wahrscheinlichkeitsth. 2, 75-84.

[24] Rényi, A. and Sulanke, R. (1964). Über die konvexe Hülle von $n$ zufällig gewählten Punkten. II. $Z$. Wahrscheinlichkeitsth. 3, 138-147.

[25] RÉNYI, A. AND Sulanke, R. (1968). Zufällige konvexe Polygone in einem Ringgebiet. Z. Wahrscheinlichkeitsth. 9, 146-157.

[26] Santaló, L. A. (1946). Sobre figuras planas hiperconvexas. Summa Bras. Math. 1, 221-239.

[27] Schneider, R. (1993). Convex bodies: The Brunn-Minkowski Theory (Encyclopedia Math. Appl. 44). Cambridge University Press.

[28] SChNeIDER, R. (2008). Recent results on random polytopes. Boll. Unione Mat. Ital. (9) 1, 17-39.

[29] SCHneIder, R. AND WeIL, W. (2008). Stochastic and Integral Geometry. Springer, Berlin.

[30] Weil, W. ANd Wieacker, J. A. (1993). Stochastic geometry. In Handbook of Convex Geometry, Vol. A, B, North-Holland, Amsterdam, pp. 1391-1438. 